Growth mechanisms and process window for InAs V-shaped nanoscale membranes on Si[001]

This content has been downloaded from IOPscience. Please scroll down to see the full text. 2013 Nanotechnology 24435603

(http://iopscience.iop.org/0957-4484/24/43/435603)

View the table of contents for this issue, or go to the journal homepage for more

Download details:

This content was downloaded by: philippec

IP Address: 211.27.6.144

This content was downloaded on 09/10/2013 at $14: 48$

Please note that terms and conditions apply. 


\title{
Growth mechanisms and process window for InAs V-shaped nanoscale membranes on $\mathrm{Si}[001]$
}

\author{
E Russo-Averchi ${ }^{1}$, A Dalmau-Mallorquí ${ }^{1}$, I Canales-Mundet ${ }^{1}$, \\ G Tütüncüoğlu ${ }^{1}$, E Alarcon-Llado ${ }^{1}$, M Heiss ${ }^{1}$, D Rüffer ${ }^{1}$, S Conesa-Boj $^{1}$, \\ P Caroff $^{2}$ and A Fontcuberta i Morral ${ }^{1}$ \\ ${ }^{1}$ Laboratoire des Matériaux Semiconducteurs, Ecole Polytechnique Fédérale de Lausanne, \\ 1015 Lausanne, Switzerland \\ ${ }^{2}$ Department of Electronic Materials Engineering, Research School of Physics and Engineering, \\ Australian National University, Canberra, ACT 0200, Australia
}

E-mail: anna.fontcuberta-morral@epfl.ch

Received 6 June 2013, in final form 4 July 2013

Published 9 October 2013

Online at stacks.iop.org/Nano/24/435603

\begin{abstract}
Organized growth of high aspect-ratio nanostructures such as membranes is interesting for opto-electronic and energy harvesting applications. Recently, we reported a new form of InAs nano-membranes grown on Si substrates with enhanced light scattering properties. In this paper we study how to tune the morphology of the membranes by changing the growth conditions. We examine the role of the V/III ratio, substrate temperature, mask opening size and inter-hole distances in determining the size and shape of the structures. Our results show that the nano-membranes form by a combination of the growth mechanisms of nanowires and the Stranski-Krastanov type of quantum dots: in analogy with nanowires, the length of the membranes strongly depends on the growth temperature and the V/III ratio; the inter-hole distance of the sample determines two different growth regimes: competitive growth for small distances and an independent regime for larger distances. Conversely, and similarly to quantum dots, the width of the nano-membranes increases with the growth temperature and does not exhibit dependence on the V/III ratio. These results constitute an important step towards achieving rational design of high aspect-ratio nanostructures.
\end{abstract}

(Some figures may appear in colour only in the online journal)

\section{Introduction}

In recent years there has been strongly increasing interest in the growth and fabrication of nanostructures, motivated by the constant decrease in the characteristic size of electronic devices [1-3] but also due to the increase of functionality of materials when reduced to the nanoscale [4-8]. Nanoscale structures are most commonly obtained in the form of nanowires. Interestingly, growth at the nanoscale allows materials to form other peculiar structures such as nanotrees [9], tripods and tetrapods [10, 11], nano-membranes, nanowalls and nanoplates [12-20]. The possibility of modifying the shape of nanostructures in bottom-up manner opens new perspectives in research areas where surfaces and shape is important such as: energy storage [21], energy generation [22, 23] and non-linear optics [24, 25]. For all this to become a reality, detailed studies on the formation mechanisms of novel nanoscale shapes are extremely important. At the same time, there has been a significant effort towards the growth of ordered nanostructures by a combination of top-down and bottom-up processes [26, 27], and towards the integration of III-V nanostructures on silicon [28-34]. The ordered growth of nanostructures on silicon opens many new perspectives as it enables to combine two very powerful platforms. The advantage of growing III-Vs in the form of a nanostructure 
is that the small footprint of nanostructures and their free-standing nature result in a very efficient strain relaxation at interfaces. As a consequence for moderate strain, coherent growth without misfit dislocation is possible [35] and when dislocations occur at larger misfit, they do not propagate in the vertical growth direction [36, 37]. A key requirement for the integration of nanostructures on silicon is the use of $\mathrm{Si}[001]$ as substrate. This is the platform in use in all the microelectronics industry, as CMOS fabrication on [110] or [111] surfaces have traditionally been hampered by their inferior gate oxide reliability. Only few groups have reported on the growth of III-V nanowires in the [001] direction [38-40], which has the additional advantage of suppressing the formation of extended planar defects and polytypism within the nanowires. We recently reported on a new form of III-V compound semiconductor nanostructures growing epitaxially as vertical wing-shaped membranes on [001] silicon substrates by solid source molecular-beam epitaxy (MBE) [24]. The nano-membranes are self-catalyzed and positioned in regular arrays of holes in a $\mathrm{SiO}_{2}$ mask. We showed that they originate from two opposite facets of a rectangular pyramidal island and extend along two opposite $\langle 111\rangle$ B directions, forming flat $\{110\}$ walls. We also showed that these membranes scatter light in an extremely efficient manner, rendering them extremely interesting for intrinsic light management applications. While in this initial work we provided a microscopic model for the formation of the membranes, no discussion on the effect of the growth conditions on the morphology of the membranes was provided. Such a study is necessary in order to complete the understanding on the growth mechanisms and to distinguish it from the growth mechanisms of nanowires.

In this work we present a detailed study on the effect of the growth conditions on morphology of the V-shaped nano-membranes. In particular, we look into the role of the $\mathrm{V} / \mathrm{III}$ ratio, substrate temperature, mask opening size and inter-hole distances in determining the size and shape of the membranes. We characterize the structures by Raman spectroscopy and we analyze the effect of the growth temperature on the crystal phase. This work opens a new path towards controlling the shape of bottom-up nanostructures.

\section{Experiments}

InAs membranes have been synthesized by molecular-beam epitaxy in a DCA P600 system. We have used [001] p-doped silicon wafers with a resistivity of $0.1-0.5 \Omega \mathrm{cm}$, patterned with holes with diameters ranging from 100 to $180 \mathrm{~nm}$ and inter-hole distances (the same are also defined as pitches in the following) ranging from 200 to $2000 \mathrm{~nm}$. The growth mask consisted of a $20 \mathrm{~nm}$ thick $\mathrm{SiO}_{2}$ layer of thermal oxide. The pattern has been predefined in a ZEP resist with electron-beam lithography and has been transferred on the oxide layer by a 12 s 7:1 buffered HF wet etch. Prior to the introduction in the MBE chamber, a further $2 \mathrm{~s}$ dip in the buffered HF solution was performed in order to guarantee a pristine surface. The substrates were subsequently degassed at $600^{\circ} \mathrm{C}$ for $2 \mathrm{~h}$ in UHV and then transferred to the growth chamber, where they were again heated to $770{ }^{\circ} \mathrm{C}$ for $30 \mathrm{~min}$ to further remove possible surface contaminants. The growth was carried out at a nominal In growth rate of $0.2 \AA \mathrm{s}^{-1}$, As 4 partial pressure between 0.2 and $1.15 \times 10^{-5}$ Torr (BEP), temperatures between 440 and $540{ }^{\circ} \mathrm{C}$, and with $7 \mathrm{rpm}$ rotation. Once the growth temperature had been reached, both sources (As and In) were opened at the same time and then switched off simultaneously at the end of the growth. The samples were then cooled down to $200^{\circ} \mathrm{C}$ and removed from the reactor.

The morphology of the samples was characterized by scanning electron microscopy (SEM). We used the imageprocessing package ImageJ [41] to automatically analyze the features shown in the pictures. Statistical information such as the length and width of the membranes are obtained by scanning top view images. The length is given by the Feret diameter, defined as the longest segment between any two points on the object (the membrane) perimeter. In the case of our membranes, this length represents the projection of the membrane's arms on the plane (001). The real length of the membranes is obtained by dividing the measured length by the cosine of the angle between the arm and the substrate $\left(34^{\circ}\right)$. The width is in turn determined by the length of a segment connecting the two points with the greatest orthogonal distance from the Feret diameter and orthogonal to their tangents. When considering the distribution of lengths in order to characterize their statistical properties, it must be taken into account that the sample may also include connected membranes [24]. In the simplest case of two connected membranes, the typical length of these structures is approximately twice the length of a membrane, in the case of three connected membranes it would be approximately thrice and so on. This requires a special care in computing the statistical properties of the sample: for example, if the multiple membranes are included in the calculation of the mean (of a single membrane) they would artificially increase the result. We handled this complication by modeling the distributions of lengths with a so-called Gaussian Mixture Model (GMM): mixture models are used in statistics when distinct subpopulations are present in a given population [42]. In our case the first subpopulation (or component, as they are also referred to) corresponds to all the single membranes (i.e. not connected with others), the second subpopulation corresponds to all the two connected membranes etc. Given that the only source of variation within each subpopulation is random, it is reasonable to model each subpopulation with a Gaussian distribution with its mean and variance. The whole population is then described as the (normalized) distribution given by the sum of all the Gaussian components (hence the name of the model). In our analysis, GMM have been fitted by using the expectation-maximization (EM) algorithm, ${ }^{3}[43]$ with a procedure available in the statistical package $R$ [44]. In

\footnotetext{
3 The EM algorithm computes efficiently the maximum likelihood estimation (MLE) in the presence of missing or hidden data by iterating two processes: the E-step, and the M-step. In the expectation, or E-step, the missing data of the complete data likelihood are estimated; in the M-step, the likelihood function (the expectation computed in the first step) is maximized. These two steps are repeated as necessary. The iteration is guaranteed to increase the likelihood and the algorithm is guaranteed to converge to a local maximum of the likelihood function.
} 
the following we will focus on the statistical properties of the single membranes component, leaving an analysis of the other components for future works.

Back-scattering micro-Raman spectra were taken on individual nano-membranes grown at temperatures from 440 to $540{ }^{\circ} \mathrm{C}$. The measurements were taken at room temperature with the $520.8 \mathrm{~nm} \mathrm{Ar-Kr}$ laser line as excitation source focused through a $63 \times$ objective (NA 0.75). The power was kept low enough to prevent heating of the structures. The crystalline structure of the InAs membranes has been analyzed with a Philips CM300 electron microscope operated at $300 \mathrm{kV}$ from the Centre Interdisciplinaire de Microscopie Électronique (CIME) at EPFL Lausanne.

\section{Temperature dependence}

Examples of InAs V-shaped membranes grown at various temperatures ranging from 440 to $540^{\circ} \mathrm{C}$ under an $\mathrm{As}_{4}$ partial pressure of $8.5 \times 10^{-6}$ Torr $(\mathrm{BEP} \mathrm{V} / \mathrm{III}=51)$ are reported in figure 1 . The scanning electron microscopy tilted images $\left(30^{\circ}\right)$ show the wing shape of the nanostructures with arms branching towards two $\langle 111\rangle \mathrm{B}$ directions [24]. The substrate temperature has a critical role on the size and the shape of the membranes. At $440{ }^{\circ} \mathrm{C}$ the tips of the membranes form flat surfaces. The section of the membranes reminds of a rectangular shape rather than a triangle. Since at these temperatures the diffusion length of In adatoms is low, the atoms depositing on the membranes cannot move easily and so they tend to accumulate in the valley formed between the arms increasing the interface height. At the higher temperatures of 480 and $500^{\circ} \mathrm{C}$, the arms of the membrane assume a triangular shape and the tip becomes sharper. It is suggested that the increased diffusion length of the In adatoms allows the atoms to reach the extreme regions of the membrane where nucleation occurs, thus explaining the wing shape and its elongation towards a sharper tip. For growth temperatures above $520^{\circ} \mathrm{C}$ the tips become rounded, the arms assume again a rectangular shape and the interface height is further reduced. This shape evolution can be understood in terms of a temperature-activated group III adatom desorption mechanism; recent reports show that indium desorption from a (111) Si surface and from a pattern containing InAs nanowires becomes relevant at substrate temperatures between 520 and $570{ }^{\circ} \mathrm{C}$ [45]. At $540{ }^{\circ} \mathrm{C}$, the nucleation yield drops since most of the In adatoms and InAs are desorbed. The nucleation is observed in only few membranes and these develop with very short and thicker arms.

Figure 2 summarizes the statistical analysis of the membrane morphology as a function of the growth temperature for a mask opening of $100 \mathrm{~nm}$ and a pitch of $1000 \mathrm{~nm}$. Similar results are obtained for other opening sizes. We choose this pitch because a pitch-less dependence on the morphology is only observed for a pitch of $1000 \mathrm{~nm}$ or higher. The dependence of the morphology on the pitch will be discussed in more detail in section 4 .

For a fixed growth time of $1 \mathrm{~h}$, the membrane length reaches values of $1500 \mathrm{~nm}$ at $480{ }^{\circ} \mathrm{C}$ while both lower and higher temperature yielded maximum lengths below $700 \mathrm{~nm}$.
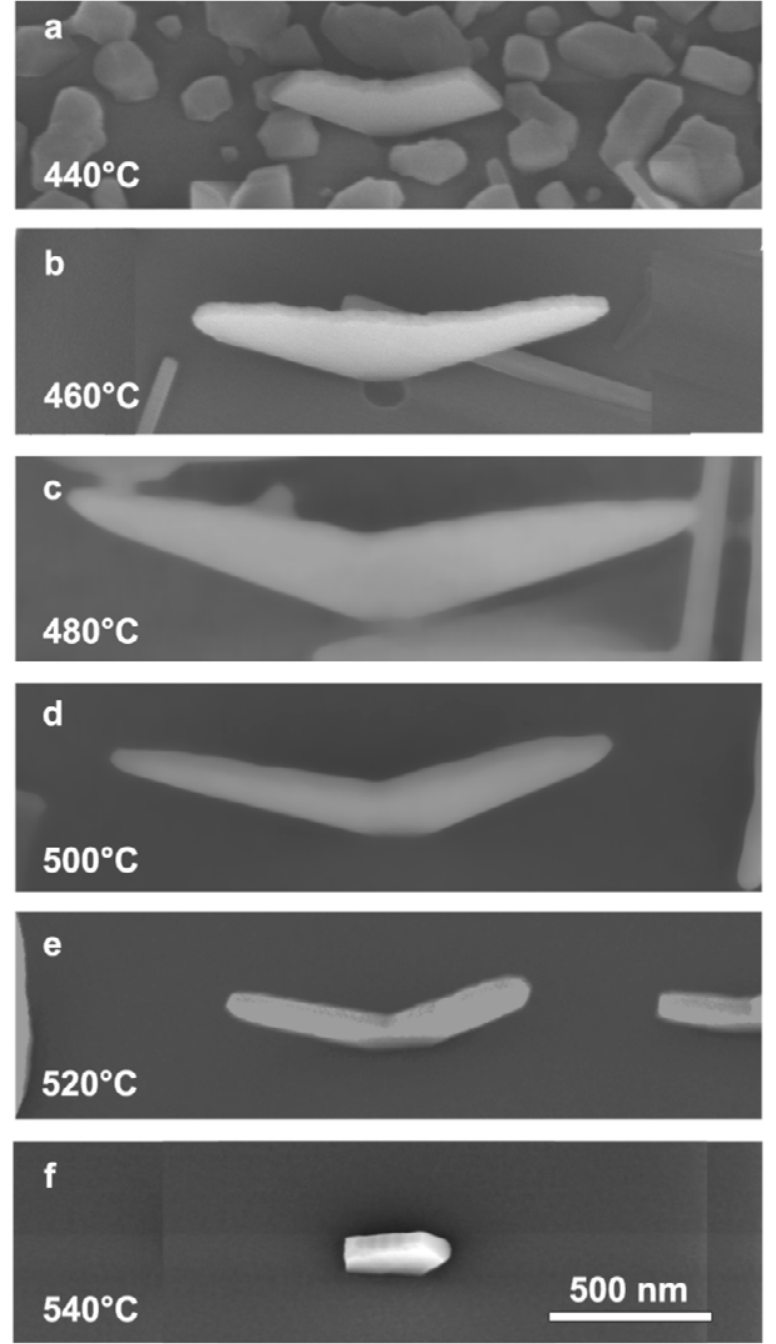

Figure 1. Scanning electron microscopy (SEM) tilted $\left(30^{\circ}\right)$ images of typical InAs membranes grown under an As partial pressure of $8.5 \times 10^{-6}$ Torr and different substrate temperatures, from $440^{\circ} \mathrm{C}$ (a) to $460{ }^{\circ} \mathrm{C}$ (b), $480{ }^{\circ} \mathrm{C} \mathrm{(c),} 500{ }^{\circ} \mathrm{C}$ (d), $520^{\circ} \mathrm{C}$ (e) and $540{ }^{\circ} \mathrm{C}$ (f). The length scale is the same for all temperatures. The images illustrate how the temperature strongly influences the length and shape of the arms of the membranes.

A similar trend has been observed several times in the growth of InAs nanowires [46-51]: the decreasing of the length by increasing the temperature above $480^{\circ} \mathrm{C}$ is related to the increased thermal decomposition rate along the $\operatorname{InAs}\langle 111\rangle$ growth direction, while the decreasing of the length by decreasing the temperature below $480^{\circ} \mathrm{C}$ can be explained by the reduced diffusivity of adatoms and hence their decreased incorporation probability at the nanostructure tip, limiting the growth along the $\langle 111\rangle \mathrm{B}$ direction. As it has been done in other works [52], we fitted the length of the membranes as a function of the inverse temperature (not shown). The results of our fit between 440 and $480^{\circ} \mathrm{C}$ show that in this range the $\langle 111\rangle$ growth rate is surface kinetically limited with an activation energy of $21.5 \pm 3.3 \mathrm{kcal} \mathrm{mol}^{-1}(0.94 \pm 0.14 \mathrm{eV})$. This value is in good agreement with the activation energy obtained by Björk et al [52] for the same material system. 

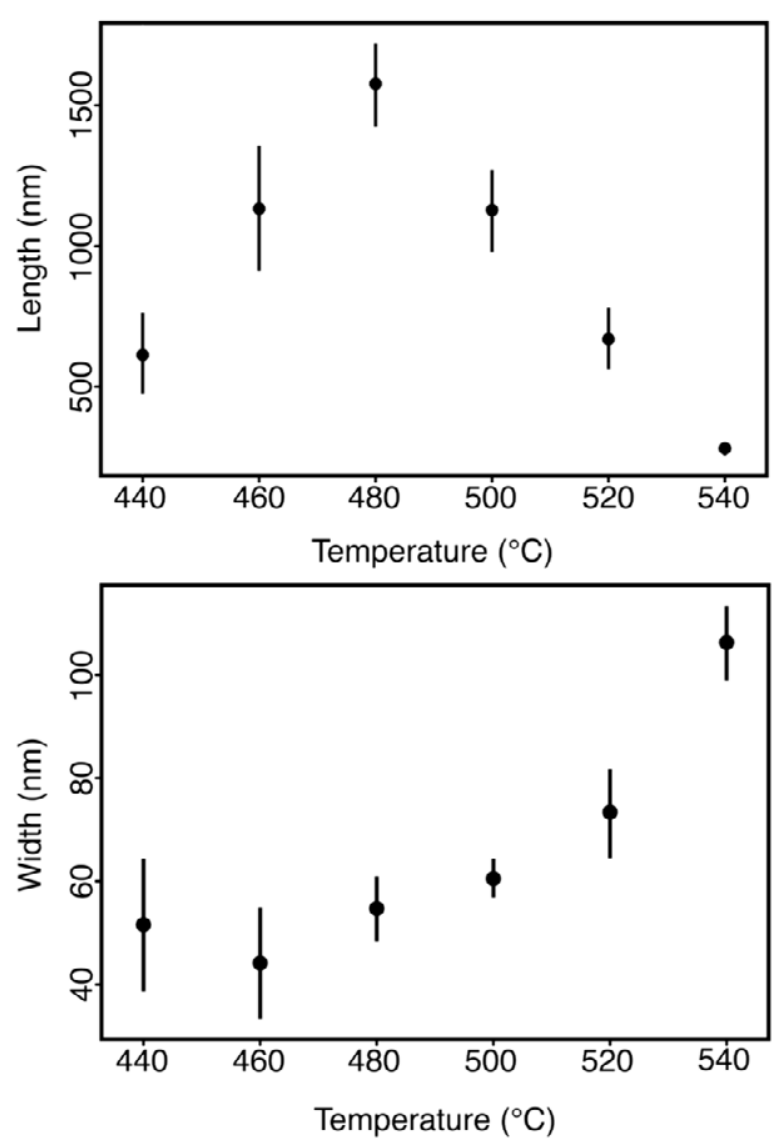

Figure 2. Temperature dependence of InAs membranes growth. The length and the width are plotted as a function of the substrate temperature, for pitch $=1000$ and for opening size $=100 \mathrm{~nm}$. Similar behavior is observed for other pitches and opening sizes. The length of the membranes first increases and then decreases with increasing temperature. In contrast, the width always increases with increasing temperature. At low temperature it reaches a plateau.

In contrast to the length, the width of the membranes increases with temperature. The variation with temperature is weak up to $480^{\circ} \mathrm{C}$, while above this value it becomes similar to what has been observed for self-catalyzed InAs nanowires [47, 53, 54], and shows a pronounced dependence on the temperature. This behavior of the membrane width could be explained by the temperature dependence of the formation of the initial nucleus from which the arms develop: a pyramidal quantum dot [24]. The arms consist of (011) and $(01 \overline{1})$ surfaces. On these planes the nucleation is relatively slow compared to $\langle 111\rangle \mathrm{B}$. Lateral growth occurs via step-flow, in agreement with the absence of tapering (homogeneous thickness). The width of the membrane is thus the distance between the two opposite $\{110\}$ planes and it is mainly determined by the width of the initial quantum dot. Indeed the width of a nucleus obtained after 5 min growth at $520^{\circ} \mathrm{C}$ is $69 \pm 9 \mathrm{~nm}$ and the width of the membranes after $1 \mathrm{~h}$ growth in the same conditions is $73 \pm 9 \mathrm{~nm}$.

A vast literature exists on the formation of InAs quantum dots on $\mathrm{Si}$ and GaAs, some of the studies including the critical growth parameters on the morphology [55-61]. For substrate temperatures above $480^{\circ} \mathrm{C}$, one observes that the quantum dot size increases with the temperature, with a scaling law similar to what we measure for the width of the membranes [60, 61]. This is explained by considering the mechanisms of quantum dot formation. After the formation of the quantum dot, the strain relaxation in the island results in a strain concentration at the island edge. The adatoms initially forming the wetting layer surface need to overcome an energy barrier $\Delta \mu$ before relax forming the island. An increase in the growth temperature leads atoms to overcome the energy barrier $\Delta \mu$ more frequently. As a consequence, the size of the quantum dot increases with the substrate temperature [62].

We turn now our attention to the evolution of the growth selectivity, namely the preferential growth of the membranes on the silicon surface of the openings rather than on the $\mathrm{SiO}_{2}$ of the growth mask, as a function of the temperature. In figure 3 we see low magnification planar view SEM images of InAs membranes grown in the conditions previously described, on patterns with opening size of $100 \mathrm{~nm}$ and inter-hole distances $400 \mathrm{~nm}$ (a)-(f) and $1800 \mathrm{~nm}(\mathrm{~g})-(\mathrm{n})$. The growth selectivity depends strongly on the growth temperature. At very low temperature, $440^{\circ} \mathrm{C}$, there is parasitic growth on the oxide in the form of InAs particles and the yield for the growth of membranes is around $20 \%$. By increasing the substrate temperature up to $480^{\circ} \mathrm{C}$, the parasitic growth decreases while at the same time the yield improves significantly. For temperatures above $480^{\circ} \mathrm{C}$, the parasitic growth on the $\mathrm{SiO}_{2}$ disappears completely, as the indium desorption rate on the oxide becomes significantly larger than the deposition rate [27, 63].

\section{Dependence on the pitch and opening size}

Figure 4 shows the length projected to the (001) plane and the width of InAs membranes grown at $\mathrm{As}_{4}$ partial pressure of $8.5 \times 10^{-6}$ and substrate temperature of $480^{\circ} \mathrm{C}$ in function of the pitch and the hole size. The length of the membrane increases with the pitch up to a size of $800 \mathrm{~nm}$ and then saturates (figure 4(a)). Growth occurs in two different regimes depending on the value of the pitch. These two regimes correspond to: (i) competitive growth regime characterized by shorter membranes for small pitches and (ii) diffusion limited or independent growth regime for longer pitches. The switch between the two regimes is determined by the indium surface diffusion length on $\mathrm{SiO}_{2}\left(\lambda_{\mathrm{SiO}_{2}}\right) \cdot \lambda_{\mathrm{SiO}_{2}}$ limits the sample area from which each membranes can collect the In species which are diffusing on the sample. For a small spacing (pitch $<2 \lambda_{\mathrm{SiO}_{2}}$ ), the indium adatoms are shared between the membranes. By increasing the spacing, the surface collection area available exclusively to each single membrane increases, resulting in a linear increase of the growth rate. In the opposite limit of a large spacing (pitch $>$ $2 \lambda \mathrm{SiO}_{2}$ ) the membranes can be treated as independent isolated islands. In this regime the surface collection area saturates and the growth rate is no longer dependent on the pitch. Similar observations have been done by other groups examining the growth of nanowires on pattern [46, 64, 65]. The width of the membranes does not show a significant dependence on the pitch (figure 4(b)). This can be related to the different 

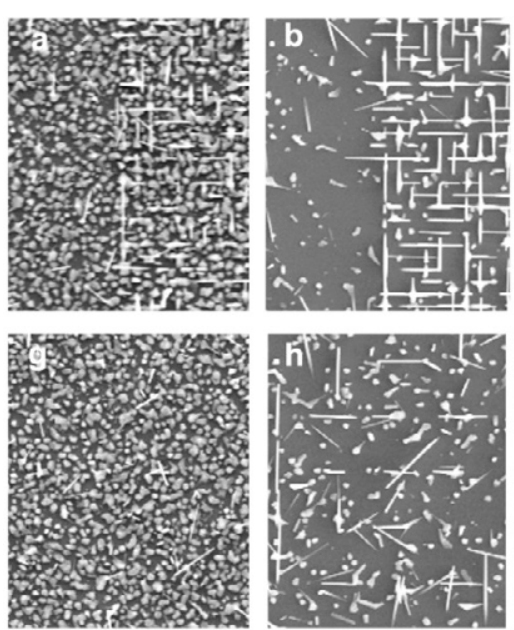
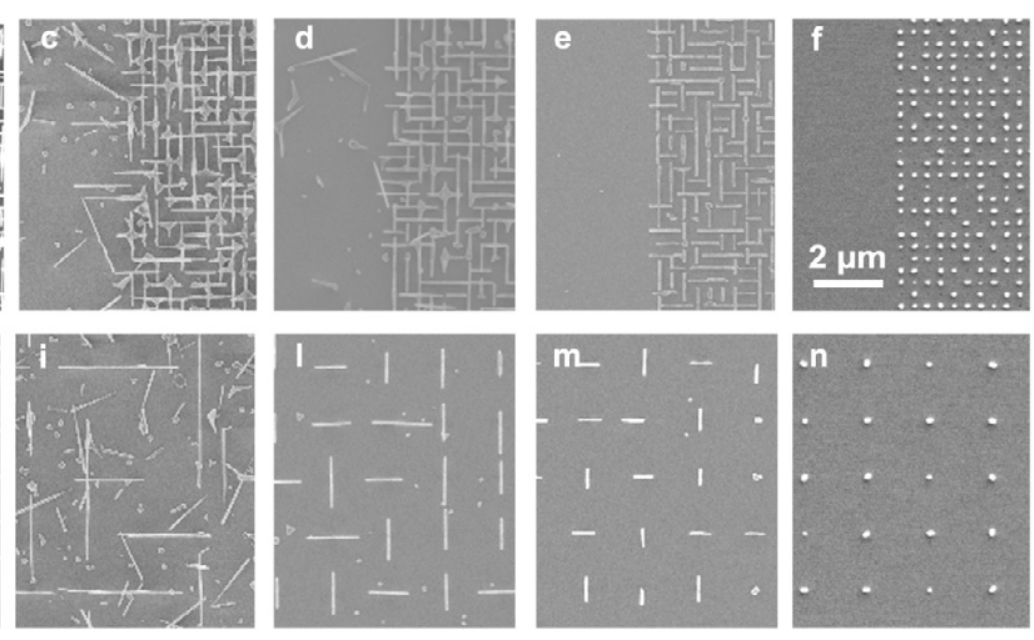

Figure 3. Planar view SEM images of InAs membranes grown under an As partial pressure of $8.5 \times 10^{-6}$ Torr and different substrate temperatures, from $440{ }^{\circ} \mathrm{C}$ (a) and (g), to $460^{\circ} \mathrm{C}(\mathrm{b})$ and (h), $480{ }^{\circ} \mathrm{C}(\mathrm{c})$ and (i), $500^{\circ} \mathrm{C}(\mathrm{d})$ and (l), $520^{\circ} \mathrm{C}(\mathrm{e})$ and (m) and $540{ }^{\circ} \mathrm{C}(\mathrm{f})$ and $(\mathrm{n})$. In these images the inter-hole distances are $400 \mathrm{~nm}$ (a)-(f) and $1800 \mathrm{~nm}(\mathrm{~g})-(\mathrm{n})$ and the opening size is $100 \mathrm{~nm}$. The scale is the same in all images. The image shows how the membrane growth selectivity strongly depends on the growth temperature and improves as the temperature increases.
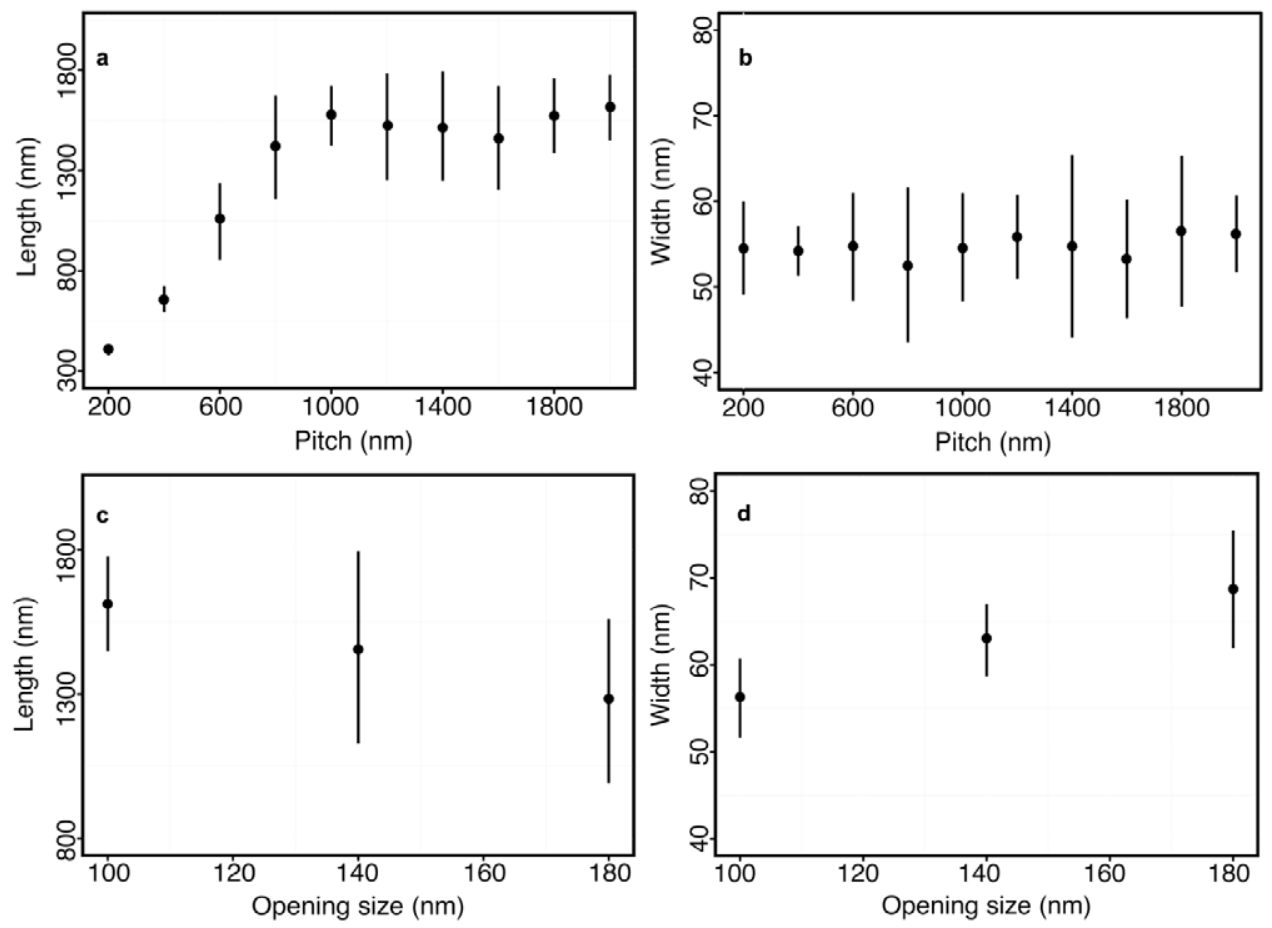

Figure 4. (a)-(b) Effect of inter-holes spacing on the length and width of InAs membranes grown under an As partial pressure of $8.5 \times 10^{-10}$ Torr and substrate temperature of $480^{\circ} \mathrm{C}$. In these images the opening size is $100 \mathrm{~nm}$. The length increases by increasing the pitch and saturates. The width does not depend on the pitch. (c)-(d) Plot of the membranes length and width as a function of the opening size (inter-hole distance is $2000 \mathrm{~nm}$ ). The length slightly decreases with the opening size while the width increases with it.

growth rate dependences of (111) B and $\{110\}$ surfaces on the growth conditions, as already shown by Björk et al [52]. In general, the length of the membranes increases with the pitch for all temperatures grown. One should note that: (i) in the competitive regime, the higher is the temperature the lower is the overall variation of the membrane length. One can also say that the variation between the length at $200 \mathrm{~nm}$ and the one at $2000 \mathrm{~nm}$ decreases by increasing the temperature; (ii) in the independent regime, the maximal length decreases by decreasing the temperature, consistently with figures 1 and 2 .

The role of the hole size is analyzed in figures 4(c) and (d): the length of the membranes slightly decreases with larger opening sizes, while their width increases. To explain this behavior we focus on the growth mechanism of the membranes. Since the InAs island is formed at the very beginning of the process, we speculate that a major 

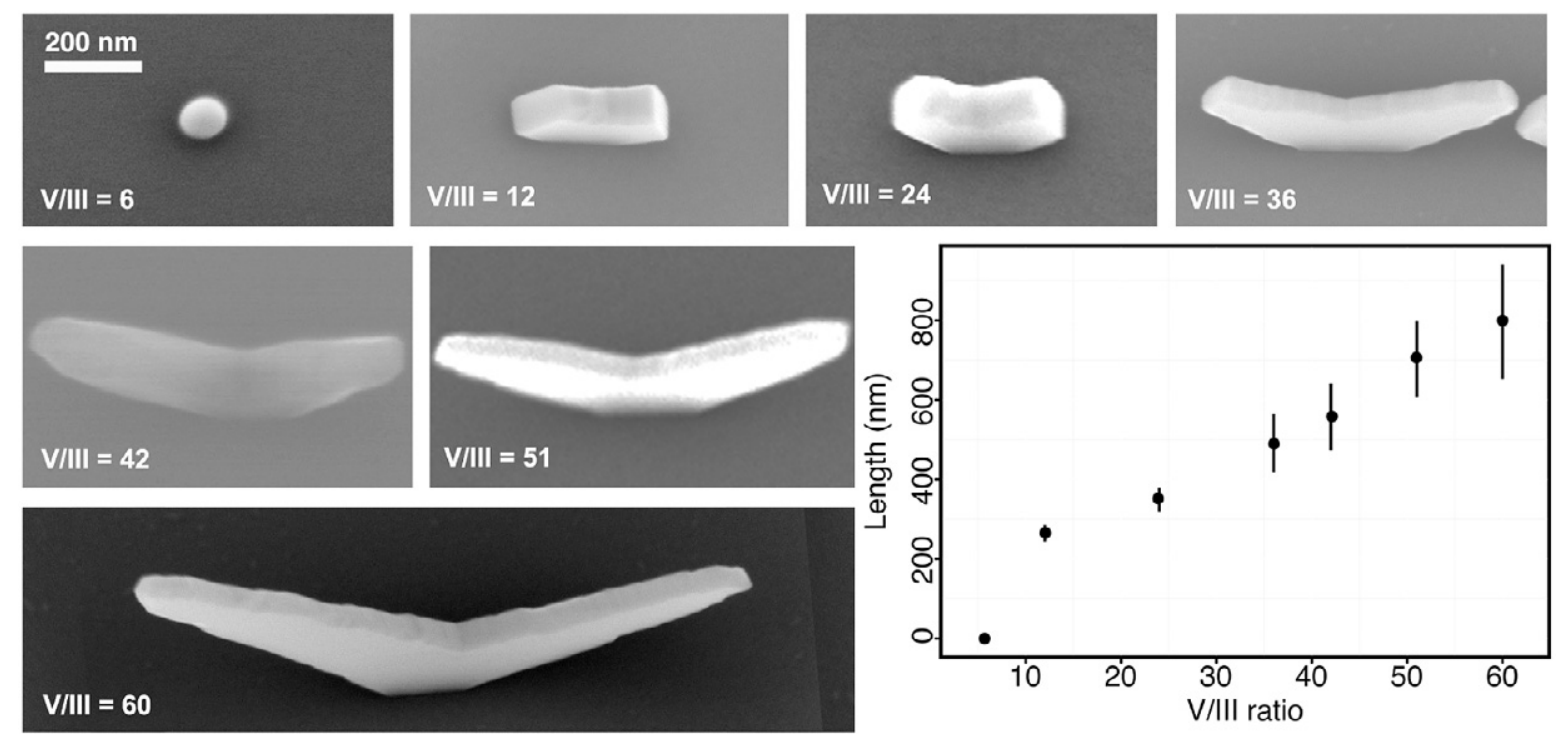

Figure 5. Tilted $\left(30^{\circ}\right)$ SEM images of InAs membranes grown at $520^{\circ} \mathrm{C}$ and under different $\mathrm{V} / \mathrm{III} \mathrm{BEP}$ ratios. The growth time is $1 \mathrm{~h}$. The length scale is the same for all pictures. At $\mathrm{V} / \mathrm{III}=6$ we do not observe any growth. Above this value the length increases with the $\mathrm{V} / \mathrm{III}$ ratio, as for group V-limited growth.

contribution to the formation of the quantum dot is given by the indium diffusing on the $\langle 001\rangle$ Si surface, i.e. the indium deposited directly into the holes. The size of the opening will thus determine the dimension of the quantum dot and in turn the width of the membrane. This has been observed by other research groups before [66, 67, 27] and our measurements confirm the findings (data not shown).

The opening size will also determine the size of the lateral facets on which the wings of the membrane grow as they scale with the size of the initial seed. One should note that the increase in width and decrease in length of the membranes with the hole size means that the total volume of the membrane is conserved and does not depend on the hole size.

\section{V/III ratio dependence}

For these experiments we have chosen $520^{\circ} \mathrm{C}$ as substrate temperature as it has shown zero parasitic growth and a good yield. In the set of high magnification $30^{\circ}$ tilted SEM images reported in figure 5, we varied the V/III ratio from 6 to 60 , by keeping the growth time for all samples equal to $1 \mathrm{~h}$. At a $\mathrm{V} / \mathrm{III}$ ratio 6 we do not observe any growth of membranes. We find only indium droplets on the surface and no island, nanowire or membrane formation. It has been seen in the past that a too low V/III ratio could lead to an indium-terminated (001) Si surface which in turn would inhibit the formation of InAs islands [68, 52]. The length of the membranes increases with the V/III ratio (graph in figure 5). We did not observe any saturation of the length for the V/III ratios used. This behavior is in agreement with group $\mathrm{V}$-limited growth mechanism. The width of the membranes is independent of the V/III ratio (data not shown).

\section{General structural characterization by Raman spectroscopy}

Having determined the effect of the growth conditions on the morphology of the V-shaped nano-membranes, we now characterize the crystal structure by Raman spectroscopy. Raman spectroscopy reports on the phonon spectrum of a material, which constitutes a fingerprint of the crystal structure. This technique has often been used in the characterization of polytypism in III-V nanostructures [69-71]. It possesses the advantage that it allows a fast non-destructive measurement and therefore the realization of a high number of measurements and statistics on a same sample. Figure 6 shows the normalized typical spectra obtained for single membranes grown at different temperatures. Most of the spectra show a main Raman feature at around $210 \mathrm{~cm}^{-1}$ and a less intense peak at $\sim 232 \mathrm{~cm}^{-1}$.

Membranes grown at the lowest temperature of $440^{\circ} \mathrm{C}$ are not considered for consistency, since the high degree of parasitic growth does not allow to optically discerning nanomembranes from the rest of deposited material. Membranes grown at mid-temperatures reveal virtually identical spectra where the frequency of the main peak corresponds to that of the $E_{2 h}$ mode of wurtzite InAs [71]. This indicates the wurtzite crystalline phase of membranes grown at such temperatures, which was further confirmed by polarization-dependent measurements on single nano-wings (not shown). In the case of membranes grown at the highest temperature $\left(540^{\circ} \mathrm{C}\right)$ the main peak clearly blue-shifts, approaching the value of the TO mode in zinc-blende InAs. This could be due to an increased zinc-blende proportion in the membranes for such temperature. In order to confirm so, high-resolution transmission electron microscopy (HRTEM) was performed. Figure $6(\mathrm{~b})$ is an HRTEM analysis on a representative InAs nano-membrane. The electron diffraction pattern of the framed area, indicates that zinc-blende crystalline structure is 
a

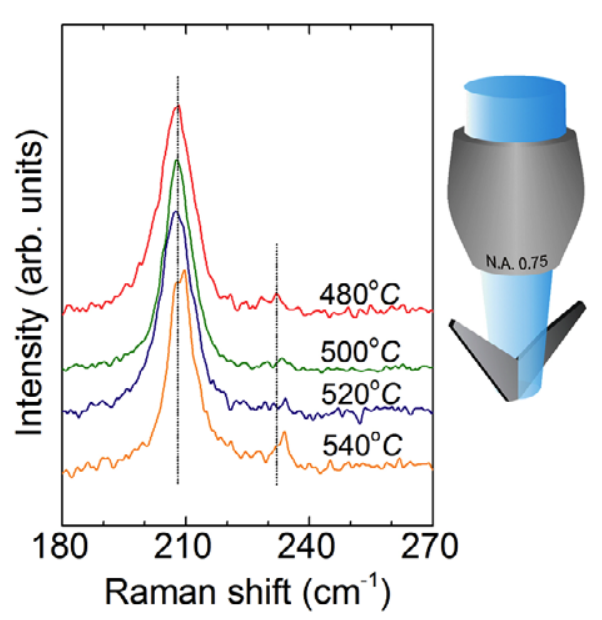

b

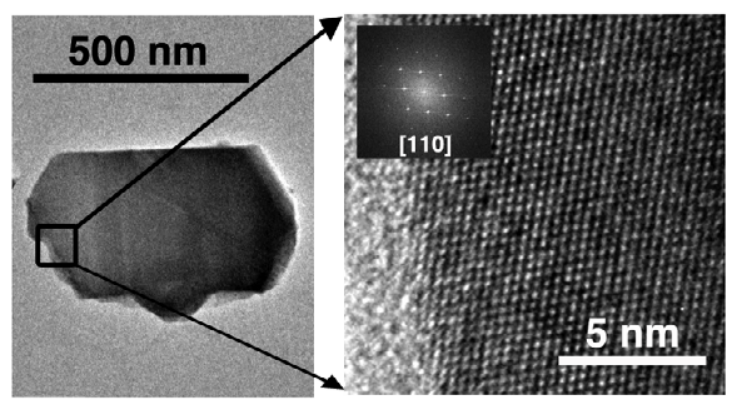

Figure 6. (a) Raman spectra obtained on single membranes as a function of the growth temperature. Dotted lines indicate the frequency position of the main phonon modes of wurtzite InAs. (b) HRTEM image of a V-shaped InAs membrane grown at $540{ }^{\circ} \mathrm{C}$ exhibiting a zinc-blende crystalline structure, as shown by the corresponding diffraction pattern, where all reflections can be indexed according to zinc-blende structure in [110] zone axis.

the main phase in the membrane wing. This constitutes the initial steps towards crystal-phase control in self-catalyzed InAs nanostructures on $\mathrm{Si}$, which is predicted to be the key development for crystal-phase engineering [72].

\section{Conclusions}

We recently reported on a new form of III-V compound semiconductor nanostructures growing epitaxially as vertical V-shaped membranes on $\mathrm{Si}[001]$. Here we studied how to control the morphology of the membranes by changing the growth conditions. In particular we have systematically examined the role of the V/III ratio, substrate temperature, mask opening size and inter-holes distances in determining the size and shape of the structures. We found that the membranes form by combining growth mechanisms of nanowires and Stranski-Krastanov type quantum dots. In analogy with nanowires, the length of the membranes strongly depends on the growth temperature and the V/III ratio; the inter-hole distance of the sample determines two different growth regimes: a competitive growth for small distances and an independent regime for larger ones. In analogy with quantum dots, the width of the membranes increases with the growth temperature and does not exhibit dependence on the V/III ratio. Our results constitute an important step towards controlling the design of these nanostructures. Lastly, we characterized the crystal structure of the membranes by Raman spectroscopy. We found that the percentage of zinc-blende material in the arms of the membranes depends on the temperature. The membranes grown at temperatures between 480 and $520^{\circ} \mathrm{C}$ have identical spectra where the frequency of the main peak corresponds to that of the $E_{2 h}$ mode of wurtzite InAs. For a temperature of $540^{\circ} \mathrm{C}$ the peak is slightly blue-shifted, towards the position of the TO mode in zinc-blende InAs and indicating an increase in the zinc-blende proportion, as confirmed by HRTEM. This result constitutes the initial steps towards crystal-phase control.

\section{Acknowledgments}

The authors thank funding from ERC through grant UpCon, FP7 project Nanoembrace, Eranet-Rus 'InCoSiN' project and SNF funding through Grants 121758/1 and 129775/1/and the NCCR QSIT. SCB thanks the Marie Heim-Vögtlin program project PMPDP2_139702 o SNF. EAL acknowledges support from Marie Curie actions. ERA thanks A Averchi for support with statistical analysis in $\mathrm{R}$ and useful discussions. PC is the recipient of an Australian Research Council Future Fellowship (project number FT120100498).

\section{References}

[1] Hu J T, Odom T W and Lieber C M 1999 Chemistry and physics in one-dimension: synthesis and properties of nanowires and nanotubes Acc. Chem. Res. 32 435-45

[2] Gudiksen M S, Lauhon L J, Wang J, Smith D C and Lieber C M 2002 Growth of nanowire superlattice structures for nanoscale photonics and electronics Nature 415 617-20

[3] Caroff P, Dick K A, Johansson J, Messing M E, Deppert K and Samuelson L 2009 Controlled polytypic and twin-plane superlattices in III-V nanowires Nature Nanotechnol. 4 50-5

[4] Cui Y and Lieber C M 2002 Functional nanoscale electronic devices assembled using silicon nanowire building blocks Science 291 851-3

[5] Mourik V, Zuo K, Frolov S M, Plissard S R, Bakkers E P A M and Kouwenhoven L P 2012 Signatures of majorana fermions in hybrid superconductor-semiconductor nanowire devices Science 25 1003-7

[6] Heiss M et al 2013 Self-assembled quantum dots in a nanowire system for quantum photonics Nature Mater. 12 439-44

[7] Hocevar M, Immink G, Verheijen M, Akopian N, Zwiller V, Kouwenhoven L and Bakkers E P A M 2012 Growth and optical properties of axial hybrid III-V/silicon nanowires Nature Commun. 31266

[8] Ohlssona B J, Björka M T, Persson A I, Thelander C, Wallenberg L R, Magnusson M H, Deppert K and Samuelson L 2002 Growth and characterization of GaAs and InAs nanowhiskers and InAs/GaAs heterostructures Physica E 13 1126-30

[9] Dick K A, Deppert K, Larsson M W, Martensson T, Seifert W, Wallenberg L R and Samuelson L 2004 Synthesis of branched 'nanotrees' by controlled seeding of multiple branching events Nature Mater. 3 380-4

[10] Dayeh S A, Yu E T and Wang D 2007 III-V nanowire growth mechanism: V/III ratio and temperature effects Nano Lett. 7 2486-90 
[11] Utama M I B, Zhang Q, Jia S K, Li D, Wang J and Xiong Q 2012 Epitaxial II-VI tripod nanocrystals: a generalization of van der Waals epitaxy for nonplanar polytypic nanoarchitectures ACS Nano 6 2281-8

[12] Liu J P, Huang X, Li Y Y, Sulieman K M and He X 2006 Hierarchical nanostructures of cupric oxide on a copper substrate: controllable morphology and wettability J. Mater. Chem. 16 4427-34

[13] Cheng W, Campolongo M J, Tan S J and Luo D 2009 Freestanding ultrathin nano-membranes via self-assembly Nano Today 4 482-93

[14] Arzt E, Gorb S and Spolenak R 2003 From micro to nano contacts in biological attachment devices Proc. Natl Acad. Sci. USA 100 10603-6

[15] Hiramatsu M, Shiji K, Amano H and Hori M 2004 Fabrication of vertically aligned carbon nanowalls using capacitively coupled plasma-enhanced chemical vapor deposition assisted by hydrogen radical injection Appl. Phys. Lett. 84 4708-10

[16] Vendamme R, Onoue S Y, Nakao A and Kunitake T 2006 Robust free-standing nanomembranes of organic/inorganic interpenetrating networks Nature Mater. 5 494-501

[17] Aagesen M, Johnson E, Sorensen C B, Mariager S O, Feidenhans'l R, Spiecker E, Nygard J and Lindelof P E 2007 Molecular beam epitaxy growth of free-standing plane parallel InAs nanoplates Nature Nanotechnol. 2 761-4

[18] Yang Y, Zhuang Y, He Y, Bai B and Wang X 2010 Fine tuning of the dimensionality of zinc silicate nanostructures and their application as highly efficient absorbents for toxic metal ions Nano Res. 3 581-93

[19] Seifert W et al 2004 Growth of one-dimensional nanostructures in MOVPE J. Cryst. Growth 272 211-20

[20] Chi C Y, Chang C C, Hu S, Yeh T W, Cronin S B and Dapkus P D 2013 Twin-free GaAs nanosheets by selective area growth-implications for defect-free nanostructures Nano Lett. ASAP. doi:10.1021/nl400561j

[21] Chan C, Peng K H, Liu G, McIlwrath K, Zhang X F, Huggins R A and Cui Y 2008 High performance lithium battery anodes using silicon nanowires Nature Nanotechnol. 3 31-5

[22] Wallentin J et al $2013 \mathrm{InP}$ nanowire array solar cells achieving $13.8 \%$ efficiency by exceeding the ray optics limit Science 339 1057-60

[23] Holm J V, Jørgensen H I, Krogstrup P, Nygård J, Liu H and Aagesen M 2013 Surface-passivated GaAsP single-nanowire solar cells exceeding $10 \%$ efficiency grown on silicon Nature Commun. 41498

[24] Conesa-Boj S et al 2012 Vertical 'III-V' V-shaped nanomembranes epitaxially grown on a patterned $\mathrm{Si}[001]$ substrate and their enhanced light scattering ACS Nano 6 10982-91

[25] Pecora E F, Walsh G F, Forestiere C, Handin A, Russo-Averchi E, Dalmau-Mallorqui A, Canales-Mundet I, Fontcuberta i Morral A and Dal Negro L 2013 Enhanced second harmonic generation from InAs nano-wings structures on silicon Nanoscale at press

[26] Plissard S, Dick K A, Larrieu G, Godey S, Addad A, Wallart X and Caroff P 2010 Gold-free growth of GaAs nanowires on silicon: arrays and polytypism Nanotechnology 21385602

[27] Plissard S, Larrieu G, Wallart X and Caroff P 2011 High yield of self-catalyzed GaAs nanowire arrays grown on silicon via gallium droplet positioning Nanotechnology 22275602

[28] Mårtensson T, Svensson C P T, Wacaser B A, Larsson M W, Seifert W, Deppert K, Gustafsson A, Wallenberg L R and Samuelson L 2004 Epitaxial III-V nanowires on silicon Nano Lett. 4 1987-90

[29] Chen R, Tran T T D, Ng K W, Ko W S, Chuang L C, Sedwick F G and Chang-Hasnain C 2011 Nanolasers grown on silicon Nature Photon. 5 170-5
[30] Bjork M T, Schmid H, Bessire C D, Moselund K E, Ghoneim H, Karg S, Lortscher E and Riel H 2010 Si-InAs heterojunction Esaki tunnel diodes with high current densities Appl. Phys. Lett. 97163501

[31] Wei W, Bao X Y, Soci C, Ding Y, Wang Z L and Wang D 2009 Direct heteroepitaxy of vertical InAs nanowires on $\mathrm{Si}$ substrates for broad band photovoltaics and photodetection Nano Lett. 92926

[32] Cirlin G E, Dubrovskii V G, Soshnikov I P, Sibirev N V, Sammsonenko Y B, Bouravleuv A D, Harmand J C and Glas F 2009 Critical diameters and temperature domains for MBE growth of III-V nanowires on lattice mismatched substrates Phys. Status Solidi (RRL) 3 112-4

[33] Krogstrup P, Popovitz-Biro R, Johnson E, Madsen M H, Nygård J and Shtrikman H 2010 Structural phase control in self-catalyzed growth of GaAs nanowires on silicon (111) Nano Lett. 10 4475-82

[34] Uccelli E et al 2011 Three-dimensional multiple-order twinning of self-catalyzed GaAs nanowires on Si substrates Nano Lett. 11 3827-32

[35] Tomioka K, Kobayashi Y, Motohisa J, Hara S and Fukui T 2009 Selective-area growth of vertically aligned GaAs and $\mathrm{GaAs} / \mathrm{AlGaAs}$ core-shell nanowires on $\mathrm{Si}(111)$ substrate Nanotechnology 20145302

[36] Balakrishnan G, Huang S, Dawson L R, Xin Y C, Conlin P and Huffaker D L 2005 Growth mechanisms of highly mismatched AlSb on a Si substrate Appl. Phys. Lett. 86034105

[37] Bessire C D, Björk M T, Schmid H, Schenk A, Reuter K B and Riel H 2011 Trap-assisted tunneling in $\mathrm{Si}$-InAs nanowire heterojunction tunnel diodes Nano Lett. 11 4195-9

[38] Krishnamachari U, Borgstrom M, Ohlsson B J, Panev N, Samuelson L, Seifert W, Larsson M W and Wallenberg L R 2004 Defect-free InP nanowires grown in [001] direction on InP(001) Appl. Phys. Lett. 852077

[39] Wang J, Plissard S, Hocevar M, Vu T T T, Zehender T, Immink G G W, Verheijen M A, Haverkort J and Bakkers E P A M 2012 Position-controlled [100] InP nanowire arrays Appl. Phys. Lett. 100053107

[40] Guo W, Banerjee A, Bhattacharya P and Ooi B S 2011 InGaN/GaN disk-in-nanowire white light emitting diodes on (001) silicon Appl. Phys. Lett. 98193102

[41] Schneider C A, Rasband W S and Eliceiri K W 2012 NIH Image to ImageJ: 25 years of image analysis Nature Methods 9 671-5

[42] Pearson K 1894 Contributions to the mathematical theory of evolution Phil. Trans. R. Soc. A 185 71-110

[43] Dempster A P, Laird N M and Rubin D B 1977 Maximum-likelihood from incomplete data via the EM algorithm J. R. Stat. Soc. B 39 1-38

[44] R Core Team 2012 A Language and Environment for Statistical Computing http://R-project.org/ (Vienna: R Foundation for Statistical Computing)

[45] Kuyyalil J, Govind, Kumar M and Shivaprasad S M 2010 Clustering and layering of In adatoms on low and high index silicon surfaces: a comparative study Surf. Sci. 604 1972-7

[46] Hertenberger S, Rudolph D, Bichler M, Finley J J, Abstreiter G and Koblmüller G 2010 Growth kinetics in position-controlled and catalyst-free InAs nanowire arrays on $\mathrm{Si}(111)$ grown by selective area molecular beam epitaxy J. Appl. Phys. 108114316

[47] Koblmüller G, Hertenberger S, Vizbaras K, Bichler M, Bao F, Zhang J P and Abstreiter G 2010 Self-induced growth of vertical free-standing InAs nanowires on $\mathrm{Si}(111)$ by molecular beam epitaxy Nanotechnology 21365602

[48] Martelli F, Rubini S, Jabeen F, Felisari L and Grillo V 2011 On the growth of InAs nanowires by molecular beam epitaxy J. Cryst. Growth 323 297-300 
[49] Tchernycheva M, Travers L, Patriarche G, Glas F, Harmand J C, Cirlin G E and Dubrovskii V G 2007 Au-assisted molecular beam epitaxy of InAs nanowires: growth and theoretical analysis J. Appl. Phys. 102094313

[50] Mandl B, Stangl J, Mårtensson T, Mikkelsen A, Eriksson J, Karlsson L S, Bauer G, Samuelson L and Seifert W 2006 $\mathrm{Au}$-free epitaxial growth of InAs nanowires Nano Lett. 6 1817-21

[51] Johansson J, Wacaser B A, Dick K A and Seifert W 2006 Growth related aspects of epitaxial nanowires Nanotechnology 17 S355-61

[52] Björk M T, Schmid H, Breslin C M, Gignac L and Riel H 2012 InAs nanowire growth on oxide-masked $\langle 111\rangle$ silicon J. Cryst. Growth 344 31-7

[53] Hertenberger S, Rudolph D, Becker J, Bichler M, Finley J J, Abstreiter G and Koblmüller G 2012 Rate-limiting mechanisms in high-temperature growth of catalyst-free InAs nanowires with large thermal stability Nanotechnology 23235602

[54] Dimakis E, Lähnemann J, Jahn U, Breuer S, Hilse M, Geelhaar L and Riechert H 2011 Self-assisted nucleation and vapor solid growth of InAs nanowires on bare Si(111) Cryst. Growth Des. 11 4001-8

[55] Zhao M, Hul'ko O, Kim H J, Liu J, Sugahari T, Shi B and Xie Y H 2004 Growth and characterization of InAs quantum dots on $\mathrm{Si}(001)$ substrates $J$. Cryst. Growth $271450-5$

[56] Cirlin G E, Petrov V N, Dubrovskii V G, Masalov S A, Golubok A O, Komyak N I, Ledentsov N N, Alferov Zh I and Bimberg D 1998 Fabrication of InAs quantum dots on silicon Tech. Phys. Lett. 24 290-2

[57] Hansen L, Bensing F and Waag A 1999 Molecular beam epitaxial growth of InAs quantum dots directly on silicon Japan. J. Appl. Phys. 38 6219-21

[58] Medeiros-Ribeiro G, Bratkovski A M, Kamins T I, Ohlberg D A A and Williams R S 1998 Shape transition of germanium nanocrystals on a silicon (001) surface from pyramids to domes Science $\mathbf{2 8 9} 353-5$

[59] Leonard D, Pond K and Petroff P M 1994 Critical layer thickness for self-assembled InAs islands on GaAs Phys. Rev. B 50 11687-92

[60] Saito H, Nishi K and Sugou S 1999 Shape transition of InAs quantum dots by growth at high temperature Appl. Phys. Lett. 74 1224-6

[61] Ren H W, Nishi K, Sugou S and Masumoto Y 1998 Size quantization in InAs/GaAs self-assembled quantum dots grown by gas-source molecular beam epitaxy Japan. $J$. Appl. Phys. 37 1548-51

[62] Chen Y and Washburn J 1996 Structural transition in large-lattice-mismatch heteroepitaxy Phys. Rev. Lett. 77 4046-9

[63] Heiss M, Riedelberger E, Bichler M, Abstreiter G and Fontcuberta i Morral A 2008 Nucleation mechanism of gallium-assisted molecular beam epitaxy growth of gallium arsenide nanowires J. Cryst. Growth 310 1049-56

[64] Borgström M T, Immink G, Ketelaars B, Algra R and Bakkers E P A M 2007 Synergetic nanowire growth Nature Nanotechnol. 2 541-4

[65] Bauer B, Rudolph A, Soda M, Fontcuberta i Morral A, Zweck J, Schuh D and Reiger E 2010 Position controlled self-catalyzed growth of GaAs nanowires by molecular beam epitaxy Nanotechnology 21435601

[66] Birudavolu S, Nuntawong N, Balakrishnan G, Xin Y C, Huang S, Lee S C, Brueck S R J, Hains C P and Huffaker D L 2004 Selective area growth of InAs quantum dots formed on a patterned GaAs substrate Appl. Phys. Lett. 85 2337-9

[67] Martin A J, Saucer T W, Rodriguez G V, Sih V and Millunchick J M 2012 Lateral patterning of multilayer InAs/GaAs(001) quantum dot structures by in vacuo focused ion beam Nanotechnology 23135401

[68] Belk J G, McConville C F, Sudijono J L, Jones T S and Joyce B A 1997 Surface alloying at InAs-GaAs interfaces grown on (001) surfaces by molecular beam epitaxy Surf. Sci. 387 213-26

[69] Zardo I, Conesa-Boj S, Peiro F, Morante J R, Arbiol J, Uccelli E, Abstreiter G and Fontcuberta i Morral A 2009 Raman spectroscopy of wurtzite and zinc-blende GaAs nanowires: polarization dependence, selection rules, and strain effects Phys. Rev. B 80245324

[70] Lopez F J, Givan U, Connel J G and Lauhon L J 2011 Silicon nanowire polytypes: identification by Raman spectroscopy, generation mechanism, and misfit strain in homostructures ACS Nano 5 8958-66

[71] Hormann N J, Zardo I, Funk S, Bolte S, Doblinger M, Koblmuller G and Abstreiter G 2011 Effects of stacking variations on the lattice dynamics of InAs nanowires Phys. Rev. B 84155301

[72] Caroff P, Bolinsson J and Johansson J 2011 Crystal phases in III-V nanowires: from random toward engineered polytypism IEEE J. Sel. Top. Quantum Electron. 72 829-46 